\title{
The role of IGFBP-5 in mediating the anti-proliferation effect of tetrandrine in human colon cancer cells
}

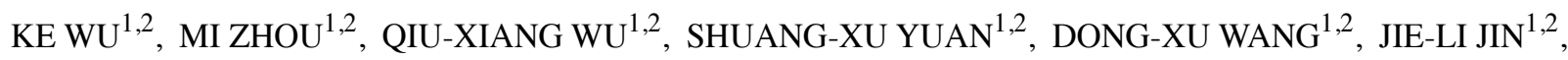 \\ JUN HUANG $^{1,2}$, JUN-QIN YANG ${ }^{1,2}$, WEN-JUAN SUN ${ }^{1,2}$, LI-HUA WAN ${ }^{3}$ and BAI-CHENG HE ${ }^{1,2}$ \\ ${ }^{1}$ Department of Pharmacology, School of Pharmacy, ${ }^{2}$ Chongqing Key Laboratory of Biochemistry \\ and Molecular Pharmacology, and ${ }^{3}$ Department of Forensic Medicine, College of Basic Medicine, \\ Chongqing Medical University, Chongqing 400016, P.R. China
}

Received October 2, 2014; Accepted November 26, 2014

DOI: $10.3892 /$ ijo.2014.2800

\begin{abstract}
Colon cancer is one of the most common malignancies, causes considerable morbidity and mortality. The current treatment for colon cancer is more modest than had been hoped. There is an urgent clinical need to explore new agents or adjuvants for colon cancer treatment. Natural products and their derivates act as one of the major source for anticancer agent. In the present study, we investigated the anti-proliferation and chemoprevention effects of tetrandrine (Tet) on colon cancer cells to uncover the possible molecular basis of this effect. We found that Tet can inhibit proliferation and induce apoptosis in LoVo cells. With dimethylhydrazine (DMH) and dextran sodium sulfate (DSS) induced colon cancer model, we found that Tet can prevent or inhibit DMH plus DSS induced aberrant crypt foci (ACF) and colon cancer formation, as well as suppress tumor growth in the xenograft colon cancer model. Tet can downregulate the expression of IGFBP-5 in LoVo cells. Exogenous expression of IGFBP-5 can attenuate the anti-cancer activity of Tet, while IGFBP-5 knockdown potentiates this effect of Tet on LoVo cells. Tet can inhibit Wnt/ $\beta$-catenin signaling transduction, which can be partly reversed by exogenous expression of IGFBP-5, but is enhanced by IGFBP-5 knockdown. Our results demonstrated that the anticancer activity of Tet in colon cancer cells may be mediated partly by downregulating the expression of IGFBP-5, thus inactivating $\mathrm{Wnt} / \beta$-catenin signaling transduction.
\end{abstract}

\section{Introduction}

Colon cancer is one of the most common malignancies and causes considerable morbidity and mortality (1). Substantial

Correspondence to: Professor Bai-Cheng He, Department of Pharmacology, Chongqing Medical University, No. 1 of Yixueyuan Road, Yuzhong, Chongqing 400016, P.R. China

E-mail: hebaicheng99@yahoo.com; 894704897@qq.com

Key words: tetrandrine, colon cancer, Wnt $/ \beta$-catenin signaling, IGFBP-5 progress has been made in colon cancer treatment and diagnosis, but the treatment of colon cancer is still challenging (2). Thus, there is an urgent clinical need to explore new agents or adjuvants for colon cancer treatment.

Natural products and their derived active components, including semi-synthetic and synthetic analogs, have served as one of the major sources for anticancer agents (3-6). A few plant derived compounds have been used for anticancer drugs for some years (7), such as vinblastine, vincristine, etoposide, teniposide, taxol and camptothecin. Hence, natural products play a major role in cancer chemotherapy.

Tetrandrine (Tet), a bis-benzylisoquinoline alkaloid, is extracted from the dried root of Stephania tetrandra S. Moore (Chinese herb hang fang ji). It has been reported that Tet can exert various effects, such as anti-inflammatory, immunosuppressive and anti-hypertensive (8-10). Also, it has been found that Tet was able to inhibit proliferation and induce apoptosis in many cancer cells, including breast, lung, colon cancer and neuroblastoma (11-14). Mechanistically, it has been reported that a few signaling pathways or critical factors are involved in the anticancer activity for Tet, such as mitogenactivated protein kinases (MAPKs) (15), Wnt/ $\beta$-catenin (13), PI3K/Akt (16) and p53 (17). We have also found that Tet can inhibit the proliferation of human colon cancer cell by targeting Wnt/ $\beta$-catenin (13), but the exact mechanism of this effect is not fully understood yet.

Insulin-like growth factor (IGF) signaling pathway plays an essential role in controlling cell differentiation, proliferation, apoptosis and aging (18). The IGF signaling is well regulated by IGF binding proteins (IGFBPs), which contains seven members (IGFBP-1 to IGFBP-7). It has been reported that IGF signaling was associated with colon cancer (19), such as IGFBP-2 and IGFBP-5 (20,21). IGFBP-5 has been reported associating with many types of cancer (21), such as breast cancer $(22,23)$, urothelial carcinoma (24), neuroblastoma (25) and osteosarcoma (26), but the role of IGFBP-5 in colon cancer remains unknown.

In the present study, we investigated the anticancer activity of Tet in human colon cancer cells, and dissected the possible molecular mechanism of this effect. Our results strongly indicate that IGFBP-5 may play an important role in colon cancer development, the anticancer activity of Tet 
in colon cancer may be mediated by inhibiting Wnt/ $\beta$-catenin signaling transduction partly through downregulating the expression of IGFBP-5.

\section{Materials and methods}

Chemicals and drug preparations. Tetrandrine (Tet) and DMH were from Sigma-Aldrich (St. Louis, MO, USA). The LoVo cell line was from the American Type Culture Collection (ATCC, Manassas, VA, USA). All antibodies were purchased from Santa Cruz Biotechnology, Inc. Cells were maintained in Dulbecco's modified Eagle's medium (DMEM) with 10\% fetal bovine serum (FBS), $100 \mathrm{U} / \mathrm{ml}$ of penicillin and $100 \mu \mathrm{g} / \mathrm{ml}$ of streptomycin at $37^{\circ} \mathrm{C}$ in $5 \% \mathrm{CO}_{2}$. Tet was prepared as previously reported for in vitro assay (27), or prepared with $0.5 \%$ carboxymethylcellulose sodium (CMC-Na) as a suspension for in vivo experiments.

Crystal violet viability assay. Crystal violet assay was conducted as previously reported (28). Briefly, LoVo cells were seeded in 24-well plates and treated with different concentrations of Tet. At 24, 48 or $72 \mathrm{~h}$ after treatment, cells were washed carefully with cold PBS $\left(4^{\circ} \mathrm{C}\right)$ and stained with $0.5 \%$ crystal violet formalin solution at room temperature for $20 \mathrm{~min}$. The stained cells were washed with water and air dried for imaging. For quantification, crystal violet in the stained cells was extracted with $1 \mathrm{ml} 20 \%$ acetic acid at room temperature for $20 \mathrm{~min}$ with gentle shaking. Absorbance at $570 \mathrm{~nm}$ was measured (29). Each assay was done in triplicate.

Reverse transcription $(R T)$ and polymerase chain reaction $(P C R)$ analysis. The total RNA were extracted with TRIzol (Invitrogen), followed by RT to generate cDNA templates. Then, the cDNA products were used for semi-quantitative PCR templates to detect the expression level of target genes. All samples were normalized with the expression level of GAPDH. The primer sequences are available upon request.

Western blot assay. Sub-confluent LoVo cells were seeded in 6-well plates, then treated with different concentrations of Tet and/or combined with corresponding recombinant adenovirus. At the scheduled time-point, cells lysates were collected and boiled for $10 \mathrm{~min}$. For nucleus protein extraction, the protein was harvested as introductions of the kit (\#78833; Thermo Fisher Scientific, Rockford, IL, USA). All samples were subjected to SDS-PAGE and transfered to polyvinylidene fluoride membranes, blotted with primary antibodies and secondary antibodies conjugated with horseradish peroxidase successively. Finally, the target bands were developed with SuperSignal West Femto Substrate (\#34095; Thermo Fisher Scientific). Each assay was done in triplicate.

Immunohistochemical staining. Tumor slides were deparaffinized and then rehydrated as previously reported (13). The deparaffinized slides were subjected to antigen retrieval and probed with an anti-IGFBP-5 antibody, or goat IgG as control, followed by incubation with biotin labled secondary antibodies and streptavidin-HRP. The target proteins were visualized by 3,3'-diaminobenzidine staining and imaged under a microscope.
Recombinant adenoviral constructs for IGFBP-5, siIGFBP-5 and GFP. The recombinant adenovirual vectors were carried out following the AdEasy system $(30,31)$. Briefly, the coding sequence (CDS) of human IGFBP-5 was amplified and sub-cloned into the shuttle vector pAdTrace-TO4, and the siRNA-knockdown oligo cassettes were cloned into the pSES1 shuttle vector (the siRNA for IGFBP-5 were designed with siDesign software). Then, the shuttle vectors were transfected in HEK293 cells to package the recombinant adenoviruses, which were designated as AdIGFBP-5, AdsiIGFBP-5 and AdGFP. All recombinant adenoviruses were tagged with green fluorescent protein (GFP) and AdGFP was used as the vector control.

Flow cytometric analysis for apoptosis and cell cycle. Sub-confluent LoVo cells were seeded in 6-well plates and treated with different concentrations of Tet and/or combined with infection of AdIGFBP-5, AdsiIGFBP5 or AdGFP for $48 \mathrm{~h}$. For apoptosis analysis, cells were harvested and washed with cold phosphate-buffered saline (PBS; $4^{\circ} \mathrm{C}$ ), followed by incubating with Annexin V-EGFP (\#KGA104; Nanjing KeyGen Biotech Co., Ltd., Nanjing, China) and propidium iodide (PI). Finally, the cells were analyzed with fluorescence activated cell sorting (FACS). For cell cycle analysis, cells were harvested and washed with PBS, fixed with cold $\left(4^{\circ} \mathrm{C}\right) 70 \%$ ethanol, washed with 50 and $30 \%$ ethanol and PBS. Finally, cells were stained with $1 \mathrm{ml}$ of $20 \mathrm{mg} / \mathrm{ml}$ PI containing RNase $(1 \mathrm{mg} / \mathrm{ml})$ in PBS for $30 \mathrm{~min}$ followed by flow cytometric analysis. Each assay was done in triplicate.

Orthotopic colon cancer model. The colon cancer model used in the present study was initialized by subcutaneous injection of DMH plus $1 \%$ DSS as previously reported (32). Briefly, Sprague-Dawely rats (weight $140 \pm 10 \mathrm{~g}$, half male and half female) were from the Animal Centre of Chongqing Medical University (Chongqing, China). All experiments were approved by the Institutional Animal Care and Use Committee (IACUC) of the Chongqing Medical University. Rats were given three subcutaneous injections of DMH $(40 \mathrm{mg} / \mathrm{kg})$ in the groin in a week (the same volume normal saline was used as control), followed by free access to drinking water containing $1 \%$ DSS for one week, and then changed to regular water and food at the beginning of the $3 \mathrm{rd}$ week. At the end of the $3 \mathrm{rd}$ week, the DMH treated rats were divided into 3 groups randomly (26 per group) and treated with different doses of Tet (25 and $50 \mathrm{mg} / \mathrm{kg}$ ) or solvent by intragastic administration, five times a week up to the 20th week. At the end of the 10th week, 10 rats of each group were sacrificed to detect the formation of ACF with methylene blue staining, and the number of ACF was counted in the colorectum, and the images analysed under a microscope. At the end of the 20th week, all rest rats were sacrificed to check the formation of colon cancer and retrieved the tumor masses for histological evaluation.

Xenograft colon cancer model. Sub-confluent LoVo cells were harvested and prepared for subcutaneous injection to the flank of athymic nude mice (female nude mice from the Animal Center of Chongqing Medical University; five mice per group). One week after injection, the animals were treated the with Tet $(40$ or $80 \mathrm{mg} / \mathrm{kg}$ ) or solvent intragastric administration. The 

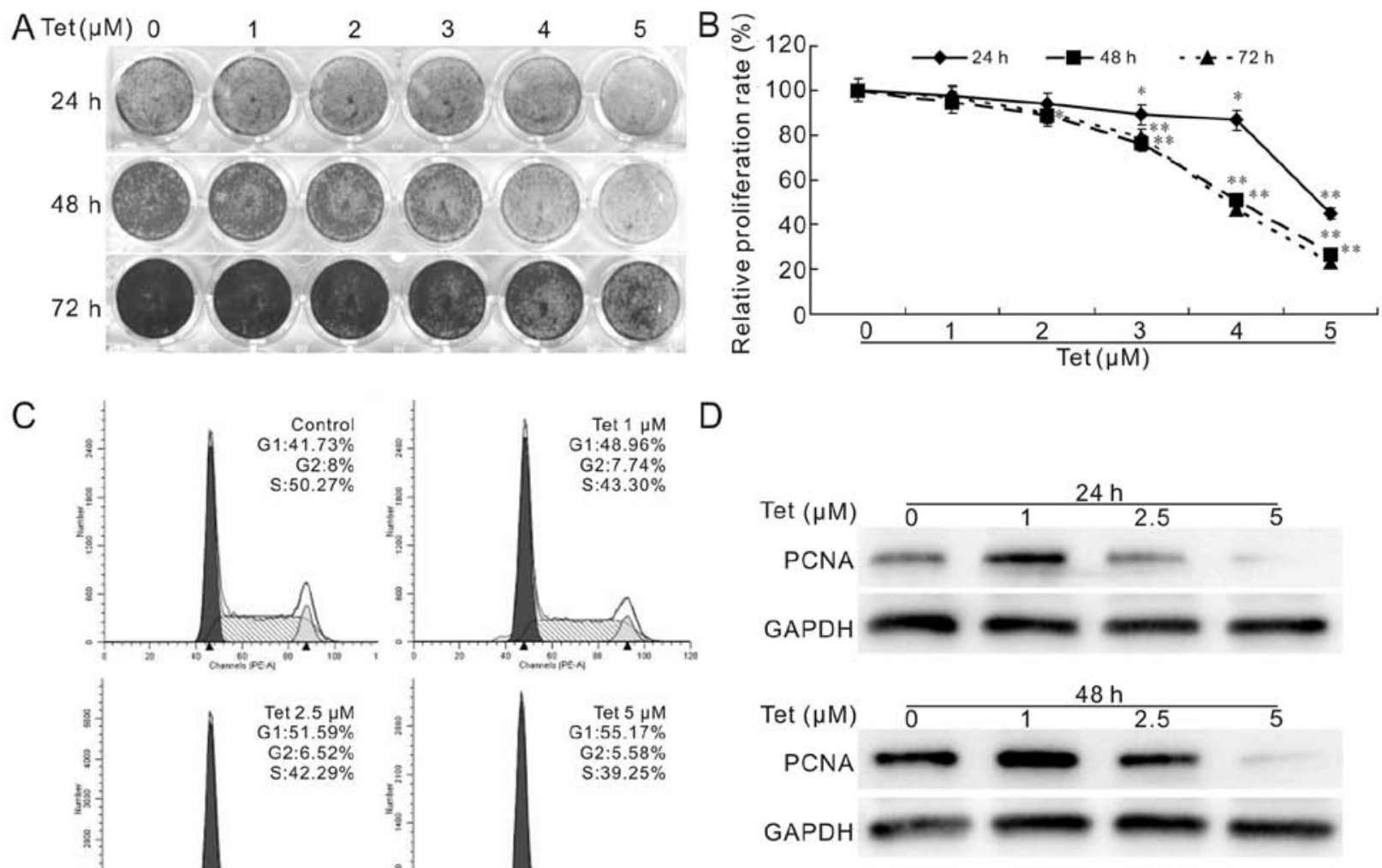

Figure 1. The effect of Tet on the proliferation in LoVo cells. (A) The crystal violet staining results show the anti-proliferation effect of Tet on LoVo cells. (B) The quantification for crystal violet staining result. ${ }^{*} \mathrm{P}<0.05$ vs. control, ${ }^{* *} \mathrm{P}<0.01$ vs. control. (C) Flow cytometric analysis results show the effect of Tet on cell cycle in LoVo cells. (D) Western blot assay results show the effect of Tet on the expression of PCNA in LoVo cells, GAPDH was used as loading control. Each condition was done in triplicate.

animals were sacrificed after 4 weeks, and the tumor masses were retrieved, fixed and paraffin-embedded. Serial sections were performed with hematoxylin and eosin (H\&E) staining for histological evaluation.

Luciferase reporter assay. Firefly reporter assay was carried out as previously reported $(30,33)$. Cells were seeded in T25 flasks and transfected with $3.0 \mu \mathrm{g}$ per flask of pTOP-Luc using Lipofectamine. Twelve hours later, cells were seeded in 24-well plates and treated with different concentrations of Tet and/or combined with corresponding recombinant adenovirus. Twenty-four hours later, cells were lysed and subjected to luciferase activity assay following the manual of the kit (E1500; Promega, Madison, WI, USA). Luciferase activity was normalized with total cellular protein concentrations of the samples. Each assay was done in triplicate.

Statistical analysis. Microsoft Excel was employed to calculate the standard deviations. The differences were analyzed using the Student's t-test.

\section{Results}

Tet inhibits the proliferation of LoVo cells. To validate whether Tet can function as a chemotherapeutic agent for human colon cancers, we first test the proliferation inhibitory effect of Tet on human colon cancer cells. The result shows that Tet inhibits the proliferation of LoVo cells effectively and concentrationdependently (Fig. 1A), even at the minimum concentration of $5 \mu \mathrm{M}$. This result is consistent with the flow cytometric assay for cell cycle analysis and western blot assay for the proliferating cell nuclear antigen (PCNA) (Fig. 1C and D). Our result in HCT116 cells was similar (13) (data not shown). These data suggest that Tet may be used as a chemotherapy agent or adjuvant for colon cancer treatment.

Tet induces apoptosis in LoVo cells. We next conducted further analyses to demonstrate whether Tet could induce colon cancer cells to undergo apoptosis. Western blot assay results show that Tet upregulates the protein level of caspase-3 and Bad, but downregulates the protein level of Bcl-2 (Fig. 2A), which is more prominent when treated the cells with Tet for $48 \mathrm{~h}$. The results of flow cytometric analysis show that the percentage of apoptotic cells increased concentration-dependently after Tet treatment (Fig. 2B), similarly to the result in HCT116 cells (13) (data not shown). Thus, these results strongly suggest that Tet can induce apoptosis in LoVo cells, a notable characteristic shared by most of the agents currently used for cancer chemotherapy.

Tet prevents DMH plus DSS induced ACF and colon cancer formation. We next investigate whether Tet could prevent the ACF and colon cancer formation induced by DMH and DSS in vivo. No ACF was found in the control group in 

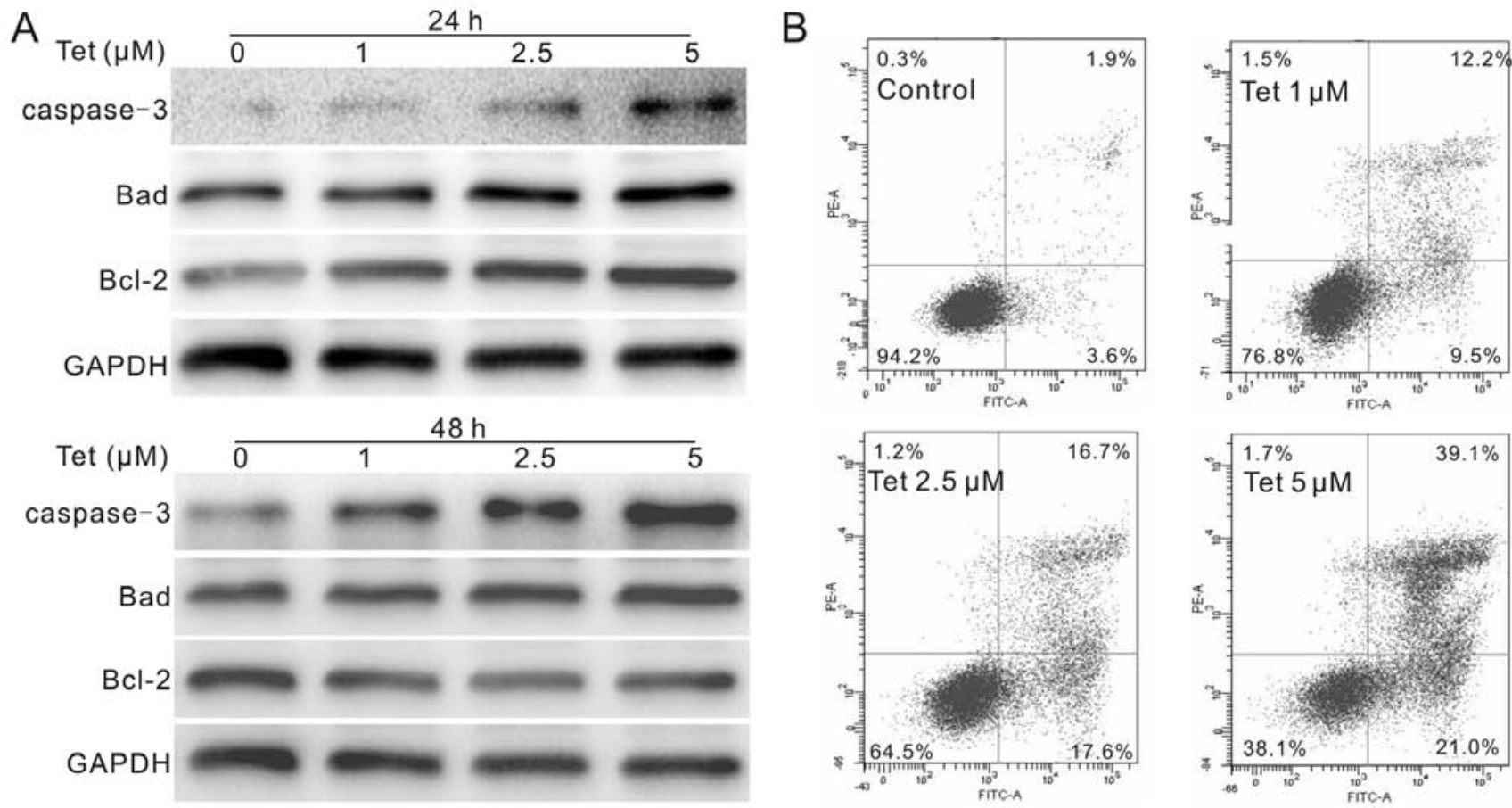

Figure 2. The effect of Tet on apoptosis in LoVo cells. (A) Western blot results show the effect of Tet on caspase-3, Bad and Bcl-2, GAPDH was used as loading control. (B) Flow cytometric analysis show the apoptosis induced by Tet. Each assay condition was done in triplicate.
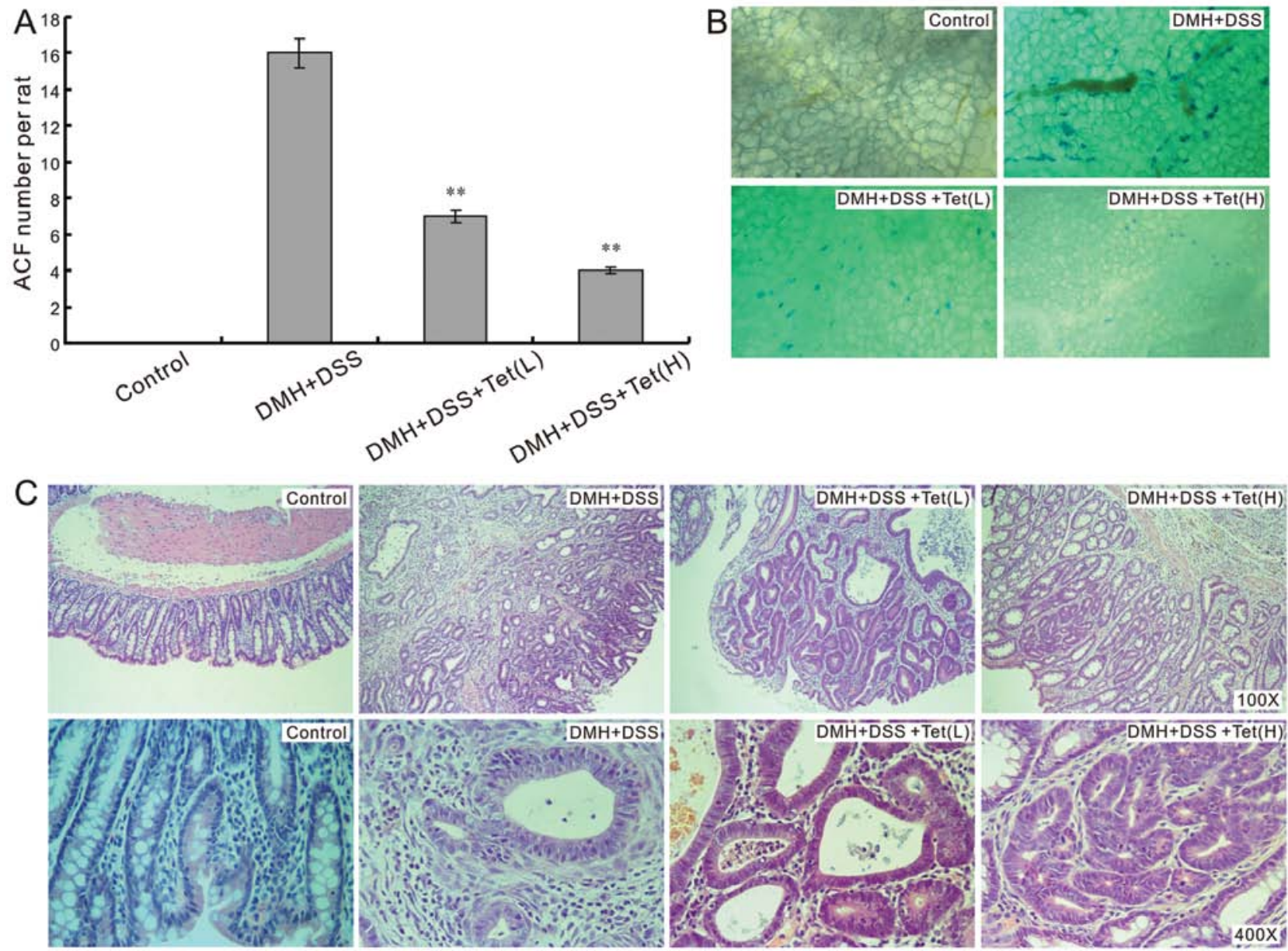

Figure 3. The effect of Tet on DMH plus DSS induced ACF and colon cancer formation. (A) The number of ACF per rat at the 10 th week. ${ }^{* *} \mathrm{P}<0.01$ vs. model group. (B) Representative images for ACF in rat colorectum (blue dots show the positive stain). (C) H\&E staining shows the colon cancer induced by DMH and DSS. 


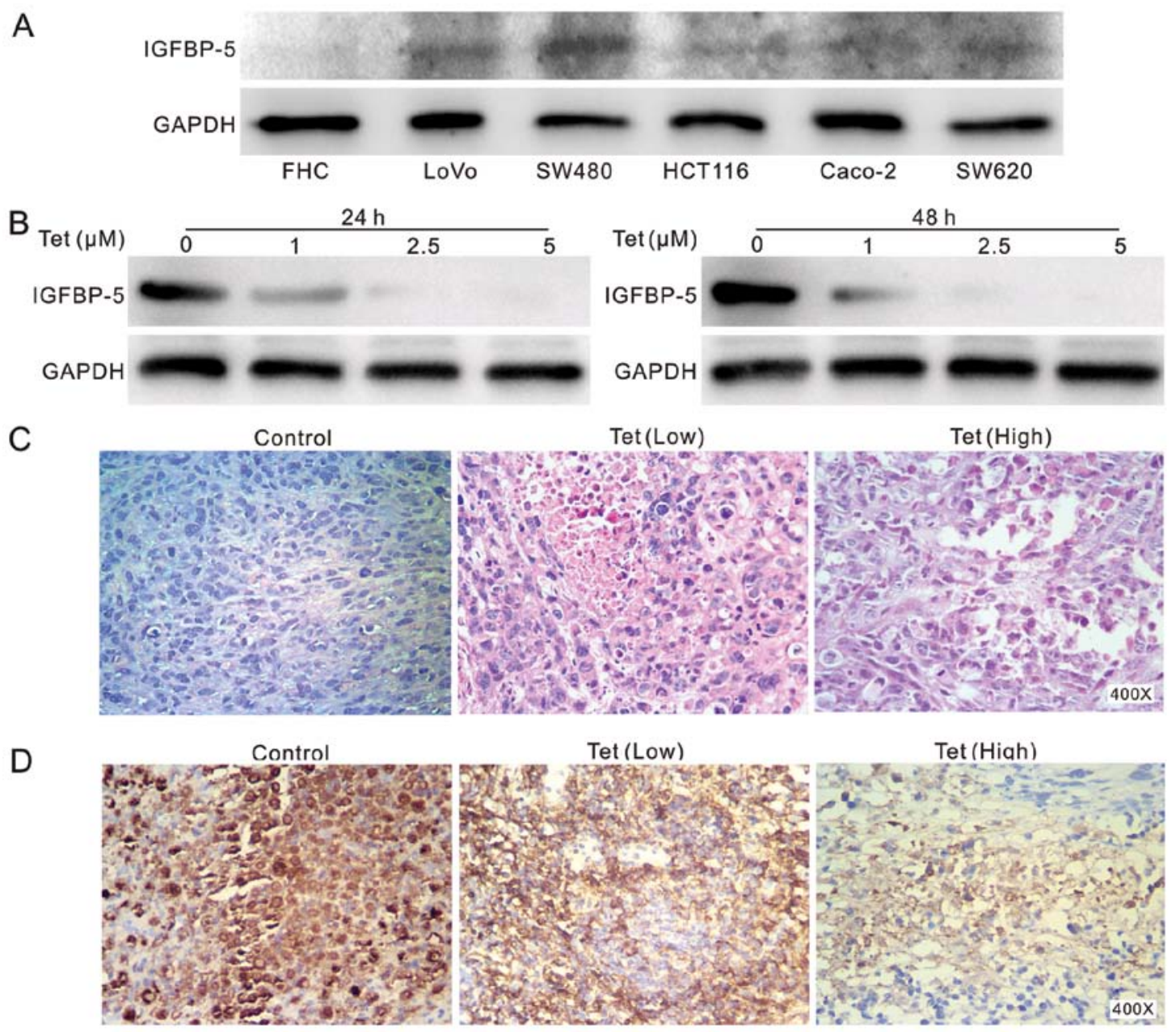

Figure 4. The effect of Tet on the expression of IGFBP-5 in colon cancer and tumor growth in xenograft colon cancer model. (A) Western blot assay shows the endogenous expression of IGFBP-5 in different colon cancer cells and FHC cells, GAPDH was used as loading control. (B) Western blot analysis shows the effect of Tet on the expression of IGFBP-5 in LoVo cells, GAPDH was used as loading control. (C) H\&E staining shows Tet inhibits the colon cancer cell growth in the xenograft tumor model. (D) Immunohistochemical staining shows the effect of Tet on the expression of IGFBP-5 in a xenograft of colon cancer.

our experiments, and the ACF number per rat in Tet treated groups was fewer than that of the model group (Fig. 3A and B). The colon cancers induced by DMH and DSS in model group was more aggressive than those of Tet treated groups, and no cancer was found in the control group. Although Tet can not block the colon cancer formation initiated by $\mathrm{DMH}$ and DSS thoroughly, it was able to attenuate the cancer grade dose-dependently (Fig. 3C). These results suggest that Tet may prevent the initiation of colon cancer.

Tet decreases the expression of IGFBP-5 and inhibits the in vivo tumor growth in the xenograft human colon cancer model. IGF plays an important role in tumorigenesis, and the inhibitor of IGF signaling has been reported as a therapeutic target for cancer $(34,35)$. IGFBP-5 is an important regulatory of IGF signaling and plays an important role in osteosarcoma (26). But, its role in colon cancer is not clear yet. Thus, we investigated whether IGFBP-5 was involved in the anti-proliferation effect of Tet in human colon cancer. We test the endogenous IGFBP-5 expression in colon cancer cell lines and fetal colon cell line (FHC), the results show that IGFBP-5 is detectable in all colon cancer cell lines and are more prominent than that in FHC cells (Fig. 4A). This result indicates that IGFBP-5 may be involved in colon cancer development. Thus, we further investigated whether Tet can regulate the expression of IGFBP-5. Western blot analysis showed that Tet can inhibit the expression of IGFBP-5 apparently in LoVo cells (Fig. 4B), and similar result was obtained in HCT116 cells (data not shown). In the xenograft colon cancer model assay, H\&E staining showed a decreased cellularity in tumor masses from Tet treated group (Fig. 4C). Immunohistochemical staining results show that Tet decreases IGFBP-5 protein level concentration-dependently in the tumor masses (Fig. 4D). These results suggest that IGBFP-5 may play an important role in colon cancer.

IGFBP-5 downregulation participates in the anti-proliferation effect of Tet on LoVo cells. As Tet can downregulate the expression of IGFBP-5, we performed further investigation to clarify how IGFBP-5 affects the anti-proliferation and apoptosis inducing effects of Tet in colon cancer cells. We constructed recombinant adenoviruses for expressing the CDS and siRNA oligo fragments of IGFBP-5, respectively. Tet treated LoVo cells, or Tet combined with AdIGFBP-5 or AdsiIGFBP-5, were collected for cell cycle and apoptosis analysis, and total protein was harvested for the detection 
A
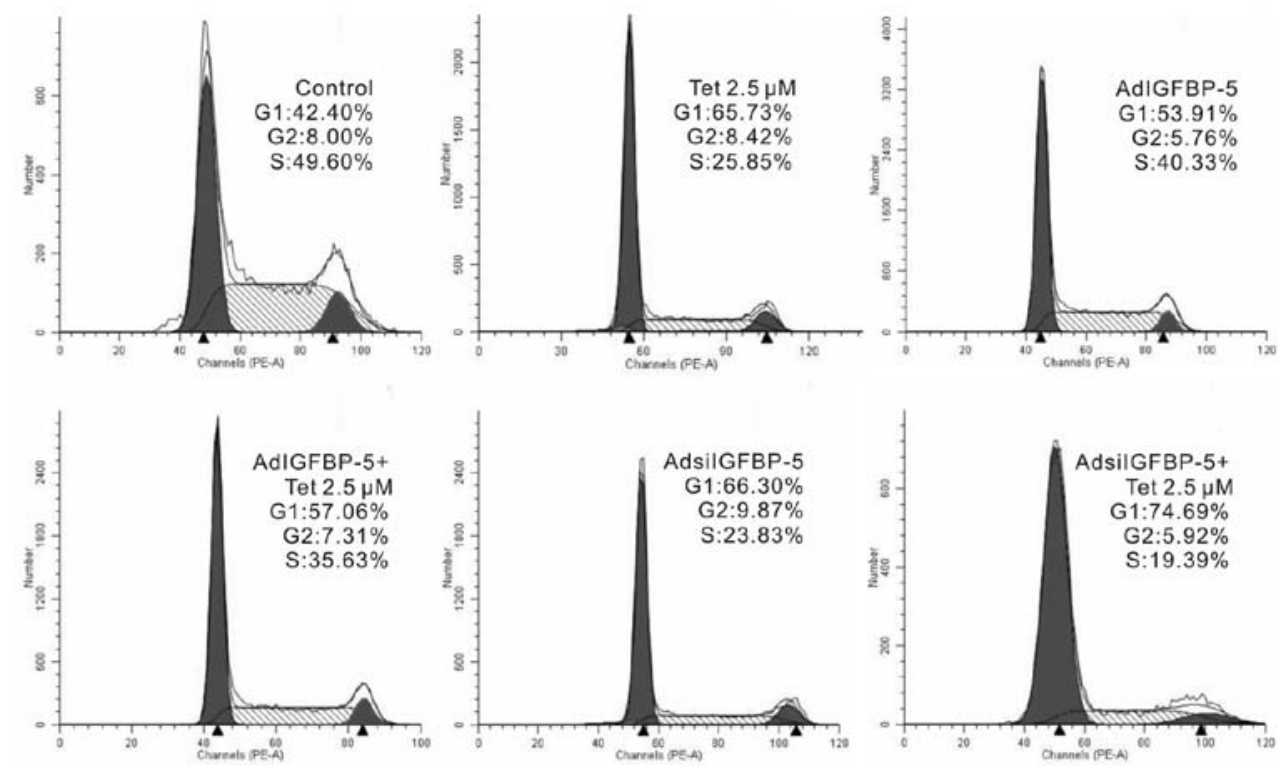

B
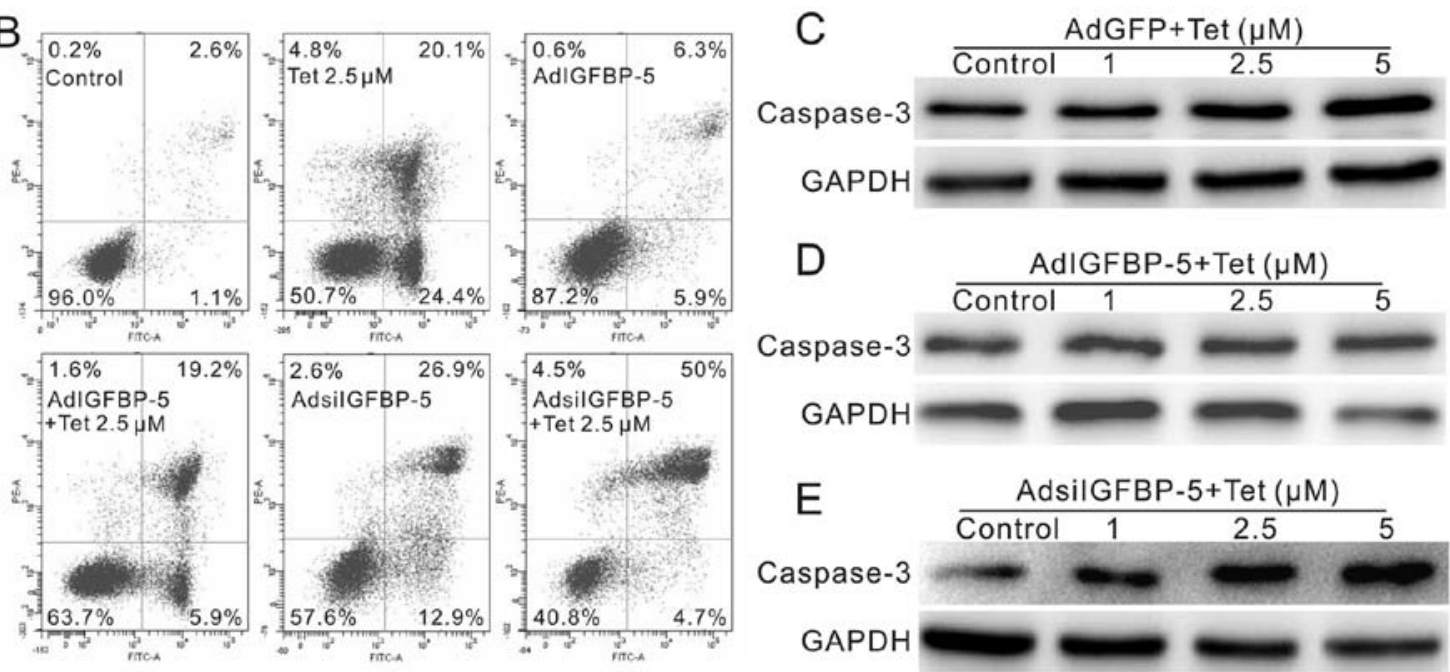

Figure 5. The effect of IGFBP-5 on the anticancer activity of Tet in LoVo cells. (A) Flow cytometric analysis shows the effect of IGFBP-5 on Tet-induced cell cycle arrest in LoVo cells. (B) Flow cytometric analysis shows the effect of IGFBP-5 on Tet-induced apoptosis in LoVo cells. (C-E) Western blot results show the effects of different IGFBP-5 expression states on Tet-induced caspase-3. GAPDH was used as loading control.

of caspase-3. The results show that exogenous expression of IGFBP-5 can reverse the cell cycle arrest effect of Tet in LoVo cells, while knockdown of IGFBP-5 enhances prominently the cell cycle arrest effect, especially the G1 phase arrest (Fig. 5A). Apoptosis analyses showed that exogenous expression of IGFBP-5 decreases the percentage of apoptotic cells induced by Tet, whereas knockdown of IGFBP-5 increases the percentage of necrotic cells (Fig. 5B). Western blot analyses indicated that Tet increases the protein level of caspase-3 (Fig. 5C), exogenous expression of IGFBP-5 has no obvious effect on the level of caspase-3 (Fig. 5D), but knockdown of IGFBP-5 enhances substantially the caspase-3 protein level (Fig. 5E). Thus, our data indicate that downregulation of IGFBP-5 may play a crucial role in Tet induced proliferation inhibition and apoptosis in LoVo cells.

IGFBP-5 affects Tet-induced downregulation of Wnt $/ \beta$-catenin signal transduction partly in LoVo cells. Wnt/ $\beta$-catenin signaling is one of the important signaling pathways for embryonic development, proliferation and differentiation. The aberrant activation of $\mathrm{Wnt} / \beta$-catenin signaling plays a critical role in the development of colorectal tumorigenesis (36). Our previous data show that Tet can target Wnt/ $\beta$-catenin signaling to inhibit the proliferation of human colon cancer cells, but the exact mechanism is unknown. Thus, we further investigate whether IGFBP-5 can affect the Tet-induced inactivation of Wnt/ $\beta$-catenin signaling transduction or not. We took pTOPluc reporter assay to test the $\mathrm{LEF} / \mathrm{Tcf}$ transcriptional activity in LoVo cells. Cells were treated with different concentrations of Tet and combined with AdGFP or AdIGFBP-5. The result shows that Tet can effectively inhibit the luciferase activities of pTOP-Luc reporter, but exogenous expression of IGFBP-5 can partly reverse this effect (Fig. 6A). Western blot analysis shows that Tet, or Tet combined with AdIGFBP-5 or AdsiIGFBP-5 has no substantial effects on $\beta$-catenin, either in cytoplasm or in the nucleus (Fig. 6B). Further analyses indicated that Tet can downregulate the expression of c-Myc, one of the important downstream targets of $\mathrm{Wnt} / \beta$-catenin signaling (37), either 

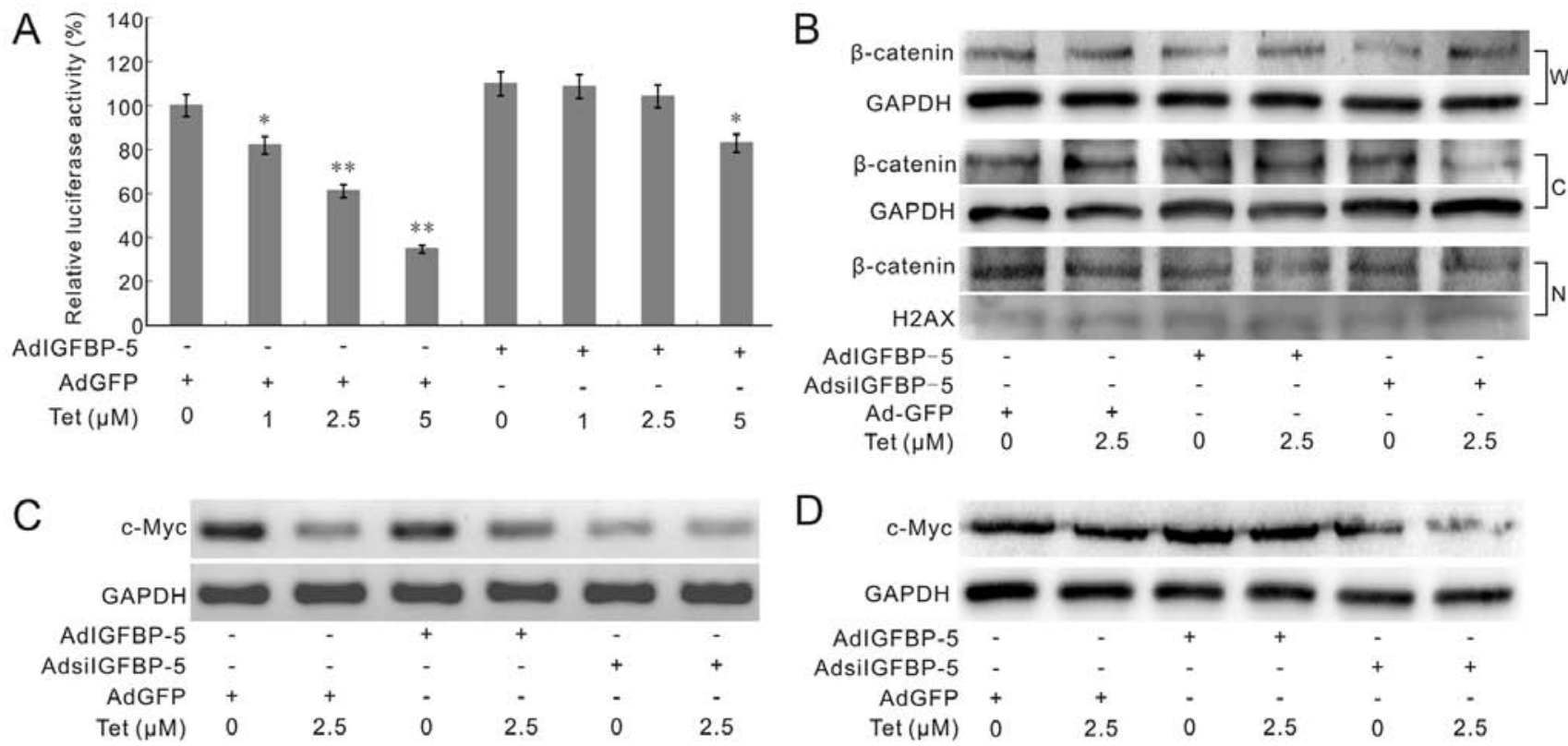

Figure 6. The effect of IGFBP-5 on Tet-induced Wnt/ $\beta$-catenin signaling inactivation in LoVo cells. (A) Luciferase reporter assay shows exogenous expression of IGFBP-5 reverses the inhibitory effect of Tet on $\beta$-catenin/Tcf transcriptional activity. ${ }^{*} \mathrm{P}<0.05$ vs. control, ${ }^{* * *} \mathrm{P}<0.01 \mathrm{vs}$. control. (B) Western blot assay shows the effect of IGFBP-5 and/or Tet on the protein level of $\beta$-catenin. (W, whole cell; C, cytoplasm; N, nucleus). GAPDH and H2AX were used as loading control, respectively. (C) RT-PCR results show the effect of IGFBP-5 and/or Tet on c-Myc expression, GAPDH was used as loading control. (D) Western blot analysis shows the effect of IGFBP-5 and/or Tet on c-Myc expression level, GAPDH was used as loading control.

the mRNA expression or protein level. This effect can also be reversed by exogenous expression of IGFBP-5, and enhanced by knockdown IGFBP5 (Fig. 6C and D). These results indicate that Tet may target Wnt/ $\beta$-catenin signaling through partly downregulating IGFBP-5, although the detailed mechanisms remain unknown.

\section{Discussion}

Tet, a bis-benzylisoquinoline alkaloid extracted from the dried root of Chinese herb hang fang $j i$, has been validated as a potential agent to inhibit proliferation and induce apoptosis in many cancer cells, such as breast, lung, colon cancer and neuroblastoma (11-14). In the present study, we demonstrated that the anticancer activity of Tet in colon cancer may be mediated by downregulating IGFBP-5 expression to inactivate the canonical Wnt signaling transduction, although the detail mechanism is still unknown.

IGF signaling pathway is a very complex system, comprising two cell surface receptors (IGF1R and IGF2R), two ligands (IGF1 and IGF2), seven binding proteins (IGFPB-1 to IGFBP-7) with high affinity to IGFs and IGFBPs degrading enzymes. The axis is very important for regulating the development and normal physiological function, including regulating cell differentiation, proliferation and apoptosis (38-41). Thus, the aberrant IGF signaling has been implicated with the development of cancer (42-45), including human colon cancer (19). IGFBP-5, as one essential member of IGF axis, has been reported implicated in cancer, but its role is controversial. IGFBP-5 inhibited breast cancer cells (22), and suppressed human osteosarcoma cell growth (26). However, exogenous IGFBP-5 also protects breast cancer from apoptosis induced by ceramide (23), prolongs breast cancer cell survival (46), and associates with metastasis and aggressiveness of breast cancer (47). Knockdown of IGFBP-5 can inhibit the proliferation and differentiation of neuroblastoma cells (25), while overexpression of IGFBP-5 indicates a poor prognosis in urothelial carcinoma (24). The diverse functions of IGFBP-5 may be dependent on the specific cellular context, cell type and the localization in cells (21), as well as the functional state of each domain (48). It is unquestionable that IGFBP-5 is implicated with cancer. However, little is known about its role in colon cancer. Thus, we investigated whether IGFBP-5 is involved in the anticancer activity of Tet in colon cancer cells. Our results show that the endogenous expression of IGFBP-5 in colon cancer cells, including LoVo, SW480, HCT116, Caco-2 and SW620, is detectable and more prominent than that of Fetal human cells (FHC), (Fig. 4A). Tet can downregulate the expression of IGFBP-5 in a concentration-dependent manner (Fig. 4B), which was confirmed by immunohistochemical staining for tumor masses (Fig. 4D). It has been reported that DMH plus DSS-induced colon cancer is characteristic of IGFBP-5 upregulation (49). Hence, we propose a hypothesis that IGFBP-5 may participate in the anticancer activity of Tet in human colon cancer. The cell cycle analysis shows that Tet can arrest cell cycle at G1 phase, and IGFBP-5 knockdown combined with Tet can enhance this effect dramatically. On the contrary, over-expression of IGFBP-5 has no substantial effect on cell cycle arrest, but it can almost reverse Tet induced cell cycle arrest in LoVo cells. These results are consistent with the apoptosis analysis (Fig. 5B and C). These data collectively support that downregulation of IGFBP-5 may play an important role in the anticancer effect of Tet in colon cancer cells.

Wnt signaling pathway is important for embryogenesis and cancer. The aberrant activation of Wnt signaling is an 
important etiological cause of colon cancer (50). For canonical Wnt pathway (also called Wnt/ $\beta$-catenin signaling pathway), $\beta$-catenin is as a critical factor. In the absence of Wnt ligands, axin, GSK-3 $\beta$ and protein APC can be assembled as a destruct complex, which can promote the proteolytic degradation of $\beta$-catenin and block the signaling transduction. In the presence of Wnt ligands, the complex will be disassembled and the $\beta$-catenin becomes stable in the cytoplasm and then translocates into the nucleus. Finally, $\beta$-catenin interacts with TCF/LEF transcription factors to regulate the expression of downstream target genes (51). Our results show that Tet can inhibit the $\beta$-catenin/TCF transcription activity, while Tet combined with exogenous expression of IGFBP-5 can partly reverse this effect (Fig. 6A). Tet can inhibit DMH plus DSSinduced colon cancer, which is characteristic of $\beta$-catenin mutation and IGFBP-5 upregulation $(49,52,53)$. Thus, the inhibitory effect of Tet on Wnt/ $\beta$-catenin signaling transduction may not fully promote the degradation of $\beta$-catenin. This was confirmed by our western blot assay, which shows that Tet, or Tet combined with IGFBP-5 overexpression or knockdown exerted no apparent effect on $\beta$-catenin protein level in the nucleus (Fig. 6B). Further analysis showed that Tet can also downregulate the mRNA and protein expression of c-Myc, an important downstream target of canonical Wnt signaling pathway (37). This can be partly reversed by exogenous expression of IGFBP-5, but enhanced by IGFBP-5 knockdown (Fig. 6C and D). These data suggest that the inhibitory effect of Tet on Wnt/ק-catenin signaling may be mediated through downregulating the expression of IGFBP-5, which may regulate the interaction of $\beta$-catenin and other important transcriptional factors to determine the expression of Wnt target genes.

Taken together, the present study strongly suggest that Tet can be used as a potent anticancer agent or adjuvant for colon cancer treatment. IGFBP-5 may be an essential factor necessary for $\beta$-catenin to interact with TCF, LEF or other essential transcription factors for $\mathrm{Wnt} / \beta$-catenin signaling transduction. The anticancer activity of Tet in colon cancer cells may be mediated partly by downregulating the expression of IGFBP-5, through which to inactivate Wnt/ $\beta$-catenin signaling transduction. Further investigations are needed to clarify the molecular mechanism of how Tet downregulates IGFBP-5, and how IGFBP-5 modulates the Wnt/ $\beta$-catenin signaling transduction, as well as the clinical significance of IGFBP-5 in colon cancer.

\section{Acknowledgements}

The authors thank Bert Vogelstein (Johns Hopkins Oncology Center, Baltimore, USA) for his kind provision of the pTOPLuc reporter vectors. This project was supported partly by the Natural Science Foundation of China (NSFC, 81071462 and 81372120 to Bai-Cheng He).

\section{References}

1. Cunningham D, Atkin W, Lenz HJ, et al: Colorectal cancer Lancet 375: 1030-1047, 2010.

2. McKeown E, Nelson DW, Johnson EK, et al: Current approaches and challenges for monitoring treatment response in colon and rectal cancer. J Cancer 5: 31-43, 2014.
3. da Rocha AB, Lopes RM and Schwartsmann G: Natural products in anticancer therapy. Curr Opin Pharmacol 1: 364-369, 2001.

4. Mann J: Natural products in cancer chemotherapy: past, present and future. Nat Rev Cancer 2: 143-148, 2002.

5. Koehn FE and Carter GT: The evolving role of natural products in drug discovery. Nat Rev Drug Discov 4: 206-220, 2005.

6. Corson TW and Crews CM: Molecular understanding and modern application of traditional medicines: triumphs and trials. Cell 130: 769-774, 2007.

7. Wang HL, Zhang XH and Chang TH: Effects of tetrandrine on smooth muscle contraction induced by mediators in pulmonary hypertension. Acta Pharmacol Sin 23: 1114-1120, 2002.

8. Shen YC, Chou CJ, Chiou WF and Chen CF: Anti-inflammatory effects of the partially purified extract of radix Stephaniae tetrandrae: comparative studies of its active principles tetrandrine and fangchinoline on human polymorphonuclear leukocyte functions. Mol Pharmacol 60: 1083-1090, 2001.

9. Li SY, Ling LH, Teh BS, Seow WK and Thong YH: Antiinflammatory and immunosuppressive properties of the bis-benzylisoquinolines: in vitro comparisons of tetrandrine and berbamine. Int J Immunopharmacol 11: 395-401, 1989.

10. Wang $\mathrm{H}$ and Chen $\mathrm{X}$ : Tetrandrine ameliorates cirrhosis and portal hypertension by inhibiting nitric oxide in cirrhotic rats. J Huazhong Univ Sci Technolog Med Sci 24: 385-388, 395, 2004.

11. Xu W, Debeb BG, Lacerda L, Li J and Woodward WA: Tetrandrine, a compound common in Chinese traditional medicine, preferentially kills breast cancer tumor initiating cells (TICs) in vitro. Cancers (Basel) 3: 2274-2285, 2011.

12. Liu W, Zhang J, Ying C, et al: Tetrandrine combined with gemcitabine and Cisplatin for patients with advanced non-small cell lung cancer improve efficacy. Int J Biomed Sci 8: 28-35, 2012.

13. He BC, Gao JL, Zhang BQ, et al: Tetrandrine inhibits Wnt/beta-catenin signaling and suppresses tumor growth of human colorectal cancer. Mol Pharmacol 79: 211-219, 2011.

14. Chen Y, Chen JC and Tseng SH: Effects of tetrandrine plus radiation on neuroblastoma cells. Anticancer Res 29: 3163-3171, 2009.

15. Wu JM, Chen Y, Chen JC, Lin TY and Tseng SH: Tetrandrine induces apoptosis and growth suppression of colon cancer cells in mice. Cancer Lett 287: 187-195, 2010.

16. Nomura M, Yamazaki R, Takaya M, et al: Inhibition of tetrandrine on epidermal growth factor-induced cell transformation and its signal transduction. Anticancer Res 27: 3187-3193, 2007.

17. Meng LH, Zhang H, Hayward L, Takemura H, Shao RG and Pommier $Y$ : Tetrandrine induces early $\mathrm{G}_{1}$ arrest in human colon carcinoma cells by down-regulating the activity and inducing the degradation of $\mathrm{G}_{1}-\mathrm{S}$-specific cyclin-dependent kinases and by inducing p53 and p21 ${ }^{\text {Cipl. }}$ Cancer Res 64: 9086-9092, 2004.

18. Kim KS, Seu YB, Baek SH, et al: Induction of cellular senescence by insulin-like growth factor binding protein-5 through a p53-dependent mechanism. Mol Biol Cell 18: 4543-4552, 2007.

19. Vanamala J, Reddivari L, Radhakrishnan S and Tarver C: Resveratrol suppresses IGF-1 induced human colon cancer cell proliferation and elevates apoptosis via suppression of IGF-1R/Wnt and activation of p53 signaling pathways. BMC Cancer 10: 238, 2010.

20. Ben-Shmuel A, Shvab A, Gavert N, Brabletz T and Ben-Ze'ev A: Global analysis of L1-transcriptomes identified IGFBP-2 as a target of ezrin and NF-kappaB signaling that promotes colon cancer progression. Oncogene 32: 3220-3230, 2013.

21. Gullu G, Karabulut S and Akkiprik M: Functional roles and clinical values of insulin-like growth factor-binding protein-5 in different types of cancers. Chin J Cancer 31: 266-280, 2012.

22. Butt AJ, Dickson KA, McDougall F and Baxter RC: Insulinlike growth factor-binding protein-5 inhibits the growth of human breast cancer cells in vitro and in vivo. J Biol Chem 278: 29676-29685, 2003.

23. Akkiprik M, Feng Y, Wang H, et al: Multifunctional roles of insulin-like growth factor binding protein 5 in breast cancer. Breast Cancer Res 10: 212, 2008.

24. Liang PI, Wang YH, Wu TF, et al: IGFBP-5 overexpression as a poor prognostic factor in patients with urothelial carcinomas of upper urinary tracts and urinary bladder. J Clin Pathol 66: 573-582, 2013.

25. Cesi V, Giuffrida ML, Vitali R, et al: C/EBP alpha and beta mimic retinoic acid activation of IGFBP-5 in neuroblastoma cells by a mechanism independent from binding to their site. Exp Cell Res 305: 179-189, 2005. 
26. Su Y, Wagner ER, Luo Q, et al: Insulin-like growth factor binding protein 5 suppresses tumor growth and metastasis of human osteosarcoma. Oncogene 30: 3907-3917, 2011.

27. Chen Y, Chen JC and Tseng SH: Tetrandrine suppresses tumor growth and angiogenesis of gliomas in rats. Int J Cancer 124: 2260-2269, 2009.

28. He BC, Chen L, Zuo GW, et al: Synergistic antitumor effect of the activated PPARgamma and retinoid receptors on human osteosarcoma. Clin Cancer Res 16: 2235-2245, 2010.

29. Ishiyama M, Tominaga H, Shiga M, Sasamoto K, Ohkura Y and Ueno K: A combined assay of cell viability and in vitro cytotoxicity with a highly water-soluble tetrazolium salt, neutral red and crystal violet. Biol Pharm Bull 19: 1518-1520, 1996.

30. He TC, Zhou S, da Costa LT, Yu J, Kinzler KW and Vogelstein B: A simplified system for generating recombinant adenoviruses. Proc Natl Acad Sci USA 95: 2509-2514, 1998.

31. Luo J, Deng ZL, Luo X, et al: A protocol for rapid generation of recombinant adenoviruses using the AdEasy system. Nat Protoc 2: 1236-1247, 2007.

32. Onose J, Imai T, Hasumura M, Cho YM and Hirose M: A new medium-term rat colon bioassay applying neoplastic lesions as endpoints for detection of carcinogenesis modifiers - validation with known modifiers. Cancer Lett 232: 272-278, 2006.

33. Zhou L, An N, Haydon RC, et al: Tyrosine kinase inhibitor STI-571/Gleevec down-regulates the beta-catenin signaling activity. Cancer Lett 193: 161-170, 2003.

34. Fidler MJ, Shersher DD, Borgia JA and Bonomi P: Targeting the insulin-like growth factor receptor pathway in lung cancer: problems and pitfalls. Ther Adv Med Oncol 4: 51-60, 2012.

35. Arcaro A: Targeting the insulin-like growth factor-1 receptor in human cancer. Front Pharmacol 4: 30, 2013.

36. Kinzler KW and Vogelstein B: Lessons from hereditary colorectal cancer. Cell 87: 159-170, 1996.

37. You Z, Saims D, Chen S, et al: Wnt signaling promotes oncogenic transformation by inhibiting c-Myc-induced apoptosis. J Cell Biol 157: 429-440, 2002

38. Gluckman P, Klempt N, Guan J, et al: A role for IGF-1 in the rescue of CNS neurons following hypoxic-ischemic injury. Biochem Biophys Res Commun 182: 593-599, 1992.

39. Valentinis B and Baserga R: IGF-I receptor signalling in transformation and differentiation. Mol Pathol 54: 133-137, 2001.

40. Fu P, Thompson JA, Leeding KS and Bach LA: Insulin-like growth factors induce apoptosis as well as proliferation in LIM 1215 colon cancer cells. J Cell Biochem 100: 58-68, 2007.
41. LeRoith D, Werner H, Beitner-Johnson D and Roberts CT Jr: Molecular and cellular aspects of the insulin-like growth factor I receptor. Endocr Rev 16: 143-163, 1995.

42. LeRoith D and Roberts CT Jr: The insulin-like growth factor system and cancer. Cancer Lett 195: 127-137, 2003.

43. Weroha SJ and Haluska P: The insulin-like growth factor system in cancer. Endocrinol Metab Clin North Am 41: 335-350, vi, 2012.

44. Wang Z, Wang Z, Liang Z, et al: Expression and clinical significance of IGF-1, IGFBP-3, and IGFBP-7 in serum and lung cancer tissues from patients with non-small cell lung cancer. Onco Targets Ther 6: 1437-1444, 2013.

45. Chen D, Siddiq A, Emdad L, et al: Insulin-like growth factorbinding protein-7 (IGFBP7): a promising gene therapeutic for hepatocellular carcinoma (HCC). Mol Ther 21: 758-766, 2013.

46. Sureshbabu A, Okajima H, Yamanaka D, et al: IGFBP5 induces cell adhesion, increases cell survival and inhibits cell migration in MCF-7 human breast cancer cells. J Cell Sci 125: 1693-1705, 2012.

47. Wang H, Arun BK, Wang H, et al: IGFBP2 and IGFBP5 overexpression correlates with the lymph node metastasis in T1 breast carcinomas. Breast J 14: 261-267, 2008.

48. Luther GA, Lamplot J, Chen X, et al: IGFBP5 domains exert distinct inhibitory effects on the tumorigenicity and metastasis of human osteosarcoma. Cancer Lett 336: 222-230, 2013.

49. Femia AP, Luceri C, Toti S, Giannini A, Dolara P and Caderni G: Gene expression profile and genomic alterations in colonic tumours induced by 1,2-dimethylhydrazine (DMH) in rats. BMC Cancer 10: 194, 2010.

50. Paez D, Gerger A, Zhang W, et al: Association of common gene variants in the $\mathrm{WNT} /$ beta-catenin pathway with colon cancer recurrence. Pharmacogenomics J 14: 142-150, 2014.

51. Kim JH, Liu X, Wang J, et al: Wnt signaling in bone formation and its therapeutic potential for bone diseases. Ther Adv Musculoskelet Dis 5: 13-31, 2013.

52. Tucker E, Buda A, Janghra B, et al: Abnormalities of the cadherin-catenin complex in chemically-induced colo-rectal carcinogenesis. Proc Nutr Soc 62: 229-236, 2003.

53. Imai T, Fukuta K, Hasumura M, et al: Significance of inflammation-associated regenerative mucosa characterized by Paneth cell metaplasia and beta-catenin accumulation for the onset of colorectal carcinogenesis in rats initiated with 1,2-dimethylhydrazine. Carcinogenesis 28: 2199-2206, 2007. 